\title{
On the Initial Pliase of Geomagnetic Storm
}

\author{
by \\ G. Ishikawa \\ Meteorological Research Institute \\ (Received September 4, 1950)
}

\begin{abstract}
A statistical survey of the sudden commencement of mag" netic storms shows two remarkable properties; in the first place, the mean frequency distribution of horizontal component $\mathrm{H}$ becomes non-symmetrical like the Pearson type, and in the second place, diurnal variation of $\mathrm{H}$ becomes W-type. After variation analysis we find that the variation in frequency distribution consists mainly of the energy variation of the corpuscular mass which is the cause of magnetic storm. This energy distribution is explained with an assumption that the sun-spot consists of a violent turbulence in which exists quasi-thermal motion by large scale masses of ionized gas which may be emitted from the sun as a unit. Wtype diurnal variation of $\mathrm{H}$ is explained as a complex phenomenon firstly with the primary field strength variation on the earth's surface produced by induced current on the front surface of corpuscuiar mass, and secondly the shielding effect of the ionosphere of the earth. The corpuscular theoy by Chapman and Ferraro is given support by our results even in numerical details, except that the conductivity of the earth's ionosphere must be by the order of $10^{-1} \sim 10^{-2}$ smaller than that expected from dynamo-theory of diurnal variation field. A rough estimation of the corpuscular mass is also attempted, and the values thus obtained are not so extravagant and inconsistent with that obtained up to this time.
\end{abstract}

\section{Introduction}

Of the various theories on the nature of geomagnetic storm, corpuscular hypothesis, which explains the storm by the electrically neutral stream of ionized particles emitted from the sun seems to be the most trustworthy for the following reasons: firstly, the time required from the passing of sun-spots across the central meridian plane to the commencement of storm is known by taking the velocity of particles to be the order of about $10^{7}-10^{8} \mathrm{~cm} / \mathrm{sec}$, the values of which are not inconsistent with the results of various authors. [1], [2], [3] Secondly, auroras, as expected from Birkeland-Störmer's theory on the basis of corpuscle hypothesis, 
are very frequently accompanied by storms. [4] [5] Such a concept has been elc b rrated by S. Chapman and V. C. A. Ferraro [6], abbreviated as (C. F.), and seems to be quite reliable, though somewhat ambiguous points still remain. Here we should like to contribute a further confirmation of their theory by producing some evidences to support it.

\section{Table I}

Group $A \cdots \cdots \cdots$.... Observed at Kakioka Magnetic Observatory.

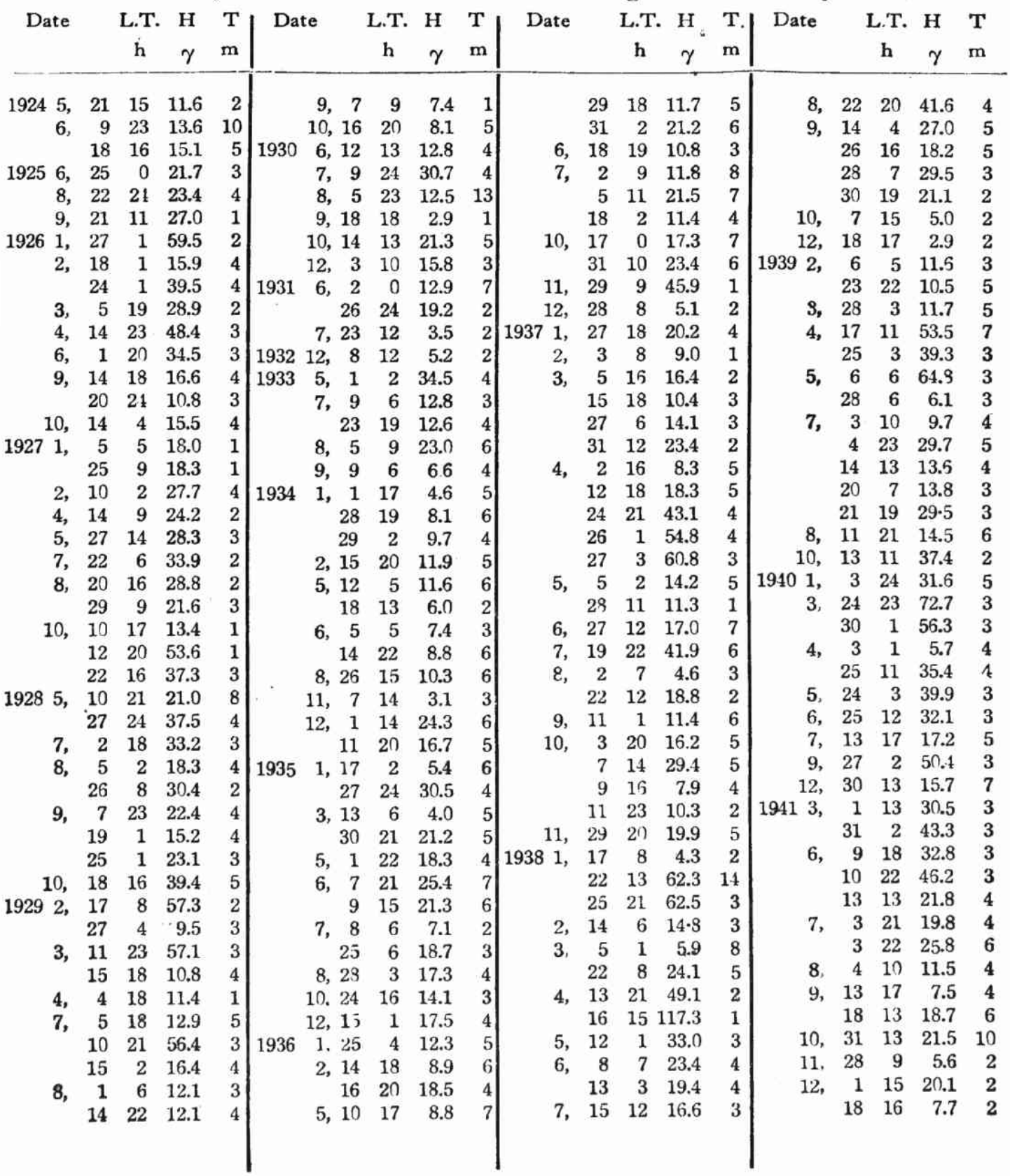




\section{Statisties of observations}

The samples used are classified into three groups, $A, B$ and $C$. Group $A$ contains 180 storms observed at Kakioka Magnetic Observatory $\left(\varphi=36^{\circ} \mathrm{N}, \lambda=140^{\circ} \mathrm{E}\right)$ from 1924 to 1941 . These values are shown in Table I. Quantitative results described below are based on this group.

Denoting the magnitude of sudden increase in horizontal intensity as $H$, we obtain a frequency distribution curve of $H$ in group $A$ as is shown in Fig. 1.

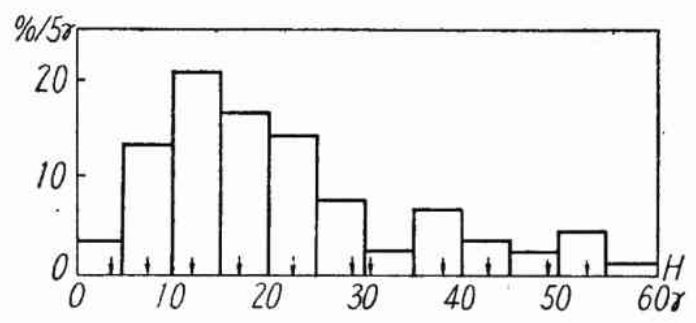

Fig. 1 The frequency distribution of $\mathbf{H}$ in group $\mathbf{A}$.

The shape of the distribution is so asymmetric that it is not adequate to carry out statistics. Hence it is necessary to transform it into normal distribution by the following variable transformation

$$
X=\log H \text {, }
$$

where $\log$ means the logarithm with base 10 . The transformed distribution curve is, as is seen in Fig. 2, fairly well represented by a normal one. Hereafter we shall discuss statistics with this transformed variable.

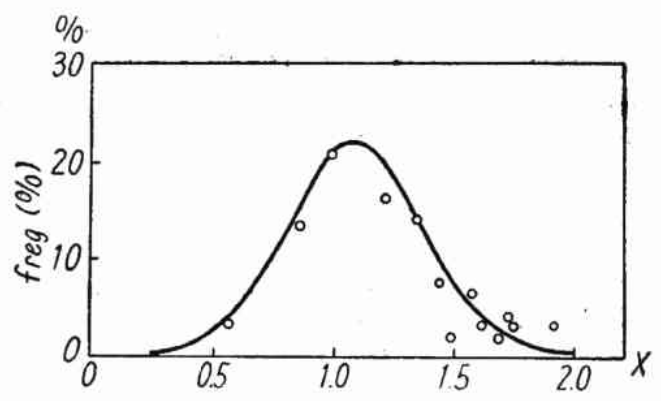

Fig. 2 The transformed frequency distribution of horizontal component.

From this we obtain the mean values respectively of $H$ and $T$, the latter means the time from sudden increase to the first apex of $H$,

$$
H=13.2 \gamma, \quad T=206 \mathrm{sec} .
$$

The quantity $X$ may show a diurnal variation, provided that the dispersion in the frequency distribution above be affected by the variation of magnetic shield- 
ing of the ionosphere. In order to facilitate this anticipation, we divide the sample $X$ into 8 classes by local time (L.T.). From the average value of $X$ in each class, we see a rather regular diurnal variation as shown in Fig. 3, the maxima exist ing at midnight and midday. We call this variation as W-type.

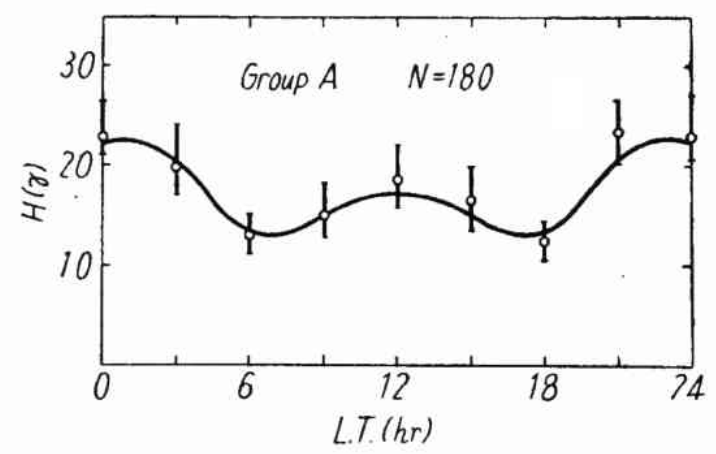

Fig. 3 The diurnal variation of $\mathrm{H}$ in group $\mathrm{A}$.

To see whether this variation is real or not, we attempt to make variation analysis, which is applied to 24 classes divided according to local time. The result in Table II shows that the unbiased estimate within groups is larger than that among $1 \mathrm{~h}$ : classes. Hence we can say from this analysis alone that the primary causes, probably the emission mechanism of the stream from the sun, should have larger variation than that of the shielding effect of the ionosphere. The frequency distribution from the primary causes alone should be obtained by subtracting the dispersion of the diurnal variation of shielding effect, which will be discussed later.

Table II

\section{Cause Degrees of freedom}

Between group means

Within groups

Total
23

156

179
Unbiased estimate
0.058
0.091
0.083

The W-type diurnal variation of $\mathrm{H}$ is still significant with confidence interval $30 \%$ as shown in Fig. 3, examined by the usual significance test.

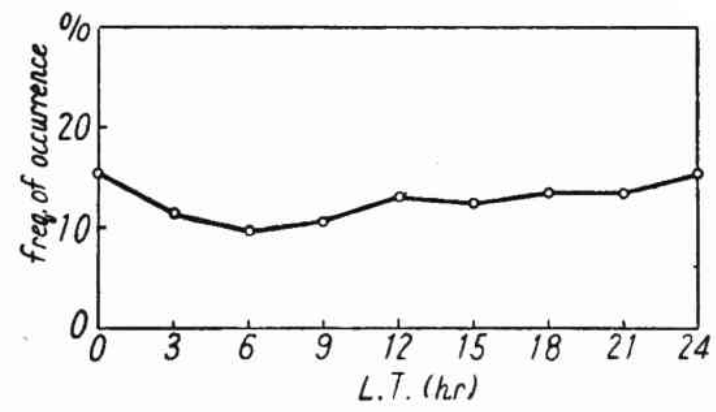

Fig. 4 The hourly frequency of occurrence of SCs at Kakioka. 
By Prof. Ferraro, these curves are not unlike those showing hourly frequency of occurrence of SCs which Dr. Parkinson and he derived in Washington. I add here the graphs of frequency of occurrence at Kakioka (Fig. 4). It seems that the minimum of occurence appears near/the sunrise and the maximum at midnight.

Further we analyzed the seasonal variation and found it significant with confidence interval $80 \%$. Unfortunately, the number of samples is too small, except in Equinox, to proceed with the quantitative discussion.

Next we discuss the $B$ and $C$ groups, which may be available for world-wide statistics. The results are, however, less reliable than the above, because of the small number of storms. Group $B$ contains 233 world-wide observed values for 14 storms ("Data for Abruptly Beginning Magnetic Disturbances No. I, II." Terr. Mag. 16, 85, 163), and group $C$ contains 209 other values obtained from reports of foreign observatories.

The positions of the observatories in these groups are shown in Table III.

Table III.

\section{Group B}

\begin{tabular}{|c|c|c|c|}
\hline No. & Observatories & Let. $(\mathrm{N})$ & Long. (E) \\
\hline 1. & Sitka, Alaska, U. S. A. & $57.1^{\circ}$ & $-135.3^{\circ}$ \\
\hline 2. & Ekaterinburg, U. S. S. R. & 56.8 & 60.6 \\
\hline 3. & Blackburn, England & 53.8 & -2.5 \\
\hline 4. & Potsdam, Germany & 52.3 & 13.0 \\
\hline 5. & Kew, England & 51.5 & -0.3 \\
\hline 6. & Greenwich, England & 51.4 & 0.0 \\
\hline 7. & Eccle, Belgique & 50.8 & 4.4 \\
\hline 8. & München, Germany & 48.1 & 11.6 \\
\hline 9. & Agincourt, Canada & 43.8 & -79.3 \\
\hline 10. & Baldwin, U. S. A. & 38.8 & -95.2 \\
\hline 11. & Cheltenham, U. S. A. & 38.7 & -76.8 \\
\hline 12. & Zi-Ka-Wei, China & 31.6 & 121.0 \\
\hline 13. & Helwan, Egypt & 29.9 & 31.3 \\
\hline 14. & Honolulu, Hawai, U. S. A. & 21.3 & -158.1 \\
\hline 15. & Bombay, India & 18.6 & 72.8 \\
\hline 16. & San Juan, Port Rico & 18.4 & -56.1 \\
\hline 17. & Batavia, Java & -5.0 & 106.7 \\
\hline 18. & Apia, Samoa & -13.8 & -171.8 \\
\hline 19. $\cdot$ & Pilar, Argentina & -31.7 & -63.9 \\
\hline \multicolumn{4}{|l|}{$\mathbf{C}$} \\
\hline 1. & Potsdam, Germany & 52.3 & 13.0 \\
\hline 2. & Cheltenham, U. S. A. & 38.7 & -76.8 \\
\hline 3. & Tucson, U. S. A. & 32.8 & -110.0 \\
\hline 4. & Zi-Ka-Wei, China & 31.6 & 121.0 \\
\hline 5. & Honolulu, Hewai, U. S. A. & 21.3 & -158.1 \\
\hline 6. & San Juan, Port Rico & 18.4 & -66.1 \\
\hline
\end{tabular}

The same statisitics as in Group $A$ result in a similar behaviour as is shown in 

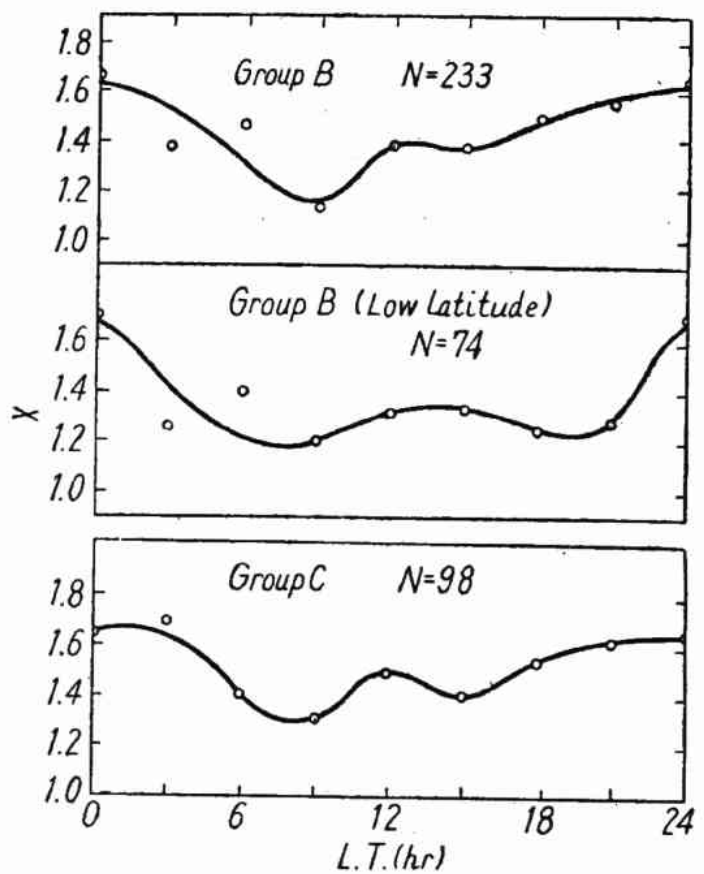

Fig. 5 The diurnal variation of $\mathbf{H}$ in groups $\mathbf{B}$ and $\mathbf{C}$.

Fig. 5, despite a rather large error.

The second figure in Fig. 5, which is obtained from the observations at latitudes lower than $35^{\circ}$, shows a lower and broader maximum at midday and seems to be not incompatible with the larger conductivity of ionosphere as will be discussed in section 6 .

\section{The discussion of the frequeney distribution of $\mathbf{H}$}

The statistics, carried out in the previous section, suggests a considerable variation in the incident ionized stream. The frequency distribution of $H$ from the primary variation alone is obtained and shown in Fig. 6.

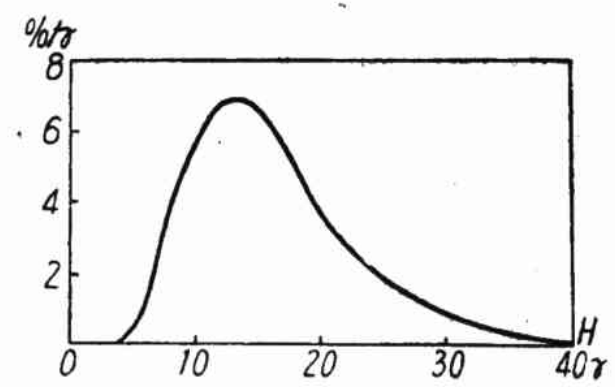

Fig. 6 The frequency distribution of $\mathrm{H}$ produced by primary causes alone.

According to (C.P.) the sudden increase is caused by the induction current produced on the front surface of the stream. The effect of this current should depend on 
the conductivity of the stream and its position relative to the earth. Since the magnetic field caused by this current is equivalent to that of the image magnet by the front surface, the variation of $H$ depends on the distance $R$ from the image magnet $C$ ' to the observation point. Here we consider the place of image magnet and consequently the induced field $H^{\prime}$ at the center of the earth $C$. Denoting $C C^{\prime}=2 Z a$, where $a$ means the radius of the earth, the induced field $H^{\prime}$ at $C$ is given by

$$
H^{\prime}=H_{0} /(2 Z)^{3},
$$

where $H_{0}$ represents the permanent magnetic field intensity at the equator of the earth's surface.

We might roughly consider that the apex point of $H$ curve occurs when the velocity of the front of the stream is reduced to $1 / 10$ of its undisturbed value. According to the approximate solution of (C.F.), $Z$, corresponding to the velocity of the front reduced to a fraction of its undisturbed value, is related with the kinetic energy density $\varepsilon$ of the stream,

$$
Z \fallingdotseq 0.324 \varepsilon^{-0.165} \text {, }
$$

where

$$
\varepsilon=\rho_{0} v_{0}^{2} / 2
$$

The quantities $\rho_{0}$ and $v_{0}$ mean the density and velocity of the stream in the undisturbed region near the earth respectively. From (3.1) and (3.2) we get

$$
H^{\prime} \fallingdotseq 1.103 \varepsilon^{0.495} \text {, }
$$

taking $H_{0}=0.3 \Gamma$. For simplicity sake we assume that

$$
H^{\prime}=\varepsilon^{1 / 2} \text {. }
$$

This is facilitated by considering that all the kinetic energy is converted into the magnetic energy. The observed field $H$ is further affected by the magnetic shielding of ionosphere by

$$
H=H^{\prime} \mid S \text {, }
$$

where $S$ is the function of the characteristic impedance of icnosphere and it will be diseussd in section 6 . Combining (3.5) with (3.4') we obtain

$$
H=\varepsilon^{1 / 2} / S \text {. }
$$

Our next task is to ascertain whether or not the observed frequency distribution is explained on the bas.s of corpuscular theory. This may be reduced to that of determination of $\varepsilon$-distribution of the stream.

How the strem can be emitted from the sun in spite of its attractive force and magnetic field has been investigated by several authors [7], [8], [9], but little discussion of the energy of the stream is presented in their papers.

Near the surface of the sun, which seems to consist of quasi-staticnary fluid system with turbulences, in which the transport of momentum or energy may be carried mainly by massive fluid elements rather than by individual atoms. These 
elements have radius of about $20 \mathrm{~km}$ and mass of about $5 \times 10^{3}$ tons, as will be shown in section 7 .

Consequently a portion of the thermal equilibrium may be made up of the virtual thermal motion of such elements, and such unit elements are considered to be emitted successively, when the turbulence becomes violent. The above concept, that the stream is emitted in masses, may be established by the observation of magnetic storms which occur intermittently though the correlation between the storm and the sun-spot, which is believed to be a violent turbulence on the sun's surface, is fairly good. In this case we can derive the energy distribution in these elements analogous to that of gas molecules. It may be assumed that the volume $V_{1}$ and density $\rho_{1}$ are constant in each element, because the kinetic energy is much more affected by the velocity cf the element than by $V_{1}$ and $\rho_{1}$. Thus we get the probability that the kinetic energy density near the sun's surface is between $\varepsilon_{1}$ and $\varepsilon_{1}+d \varepsilon_{1}$,

$$
f_{1}\left(\varepsilon_{1}\right) d \varepsilon_{1}=\frac{2}{\sqrt{ } \pi} \varepsilon_{1 m}^{-\frac{3}{2}} \sqrt{\varepsilon_{1}} \exp \left(-\frac{\varepsilon_{1}}{\varepsilon_{1 m}}\right) d \varepsilon_{1},
$$

where $\varepsilon_{1 m}$ means the most probable energy density of corpuscular mass. The energy of an emitted mass increases with radiation pressure, and is reduced with selfdiffusion. Consequently, the energy density near the earth is given by

$$
\varepsilon=\delta\left(\varepsilon_{1}+\varepsilon_{2}\right),
$$

where $\varepsilon_{2}$ is the added energy density due to radiation pressure and gravity potential, and $\delta=\rho_{0} / \rho_{1}$. Substituting (3.6) and (3.8) in (3.7), probability density function of $H$ is given by

$$
F(H)=\frac{4}{\sqrt{\pi} H^{3}{ }_{1 m}} H \sqrt{H^{2}-H_{2}^{2}} \exp \left(-\frac{H^{2}-H_{2}^{2}}{H_{1 m^{2}}}\right),
$$

where

$$
H_{l}=H_{i}^{\prime} / S=\sqrt{\delta \varepsilon_{i}} / S
$$

means the magnetic field intensity at $C$ caused by the energy density $\delta \varepsilon_{i}$ of the corpuscular mass in the presence of ionosphere. But it is not accurate to say that $F(H)$ agrees with the mean frequency distribution, observed at the earth's surface. For the mean frequency distribution, obtained with the observed value of $H$, is in a complicate relation to the distance $R$, consequently on the mean value of energy density $\varepsilon$. We shall postpone this discussion for section 6 . Here we put

$$
H_{c}=H / \cos \varphi,
$$

assuming that $Z$ is larger enough than the unit, and $\phi$ represents the latilude at the observed point. From Fig. 6 we get

$$
\left(H_{2}\right)_{a t c} \simeq 6 \gamma \text {. }
$$

Fig. 7 represents $F(H)$ for various values of $H_{1 m}$. 


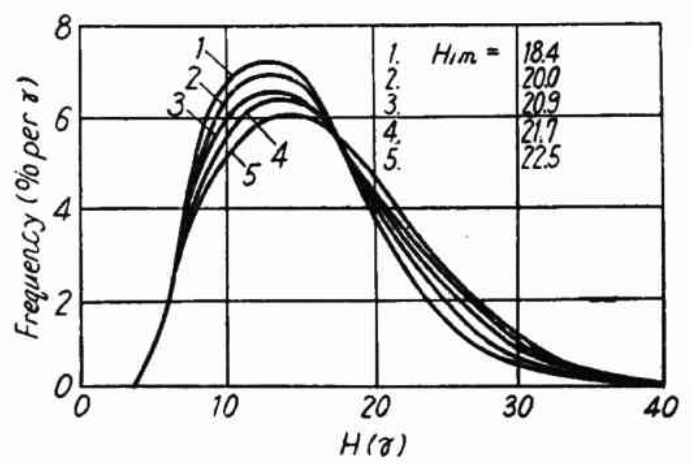

Fig. 7 The calculated probability density function of $\mathbf{H}$ for various cases.

Here

$$
H_{1 m}=20 \gamma
$$

is the best approximation to the real value. Corresponding to (3.12) and (3.13) we obtain the energy densities

$$
\begin{array}{lll}
\varepsilon_{2}=3.6 \times 10^{-9} & S^{2} / \delta & \mathrm{erg} / \mathrm{cc}, \\
\varepsilon_{1 m}=4.0 \times 10^{-8} & S^{2} / \delta & \mathrm{erg} / \mathrm{cc} .
\end{array}
$$

\section{The spatial distribution of the primary disturbing field}

In order to explain the second noticeable point, W-type diurnal variation as is seen in our statistics, we pay attention to the following two phenomena. The first is the variation of the magnetic field at the ground surface of the earth caused by the relative position against the image magnet, that is, the spatial distribution of the primary disturbing field; the second is the magnetic shielding effect of the icnosphere. These two causes, when superimposed, will explain the observed diurnal variation. In this section we consider the former problem, restricting our treatment in the case of Equinox for simplicity's sake.

The magnetic potential of the image magnet is expressed by the polar coordinate $(r, \phi, \lambda)$ with the origin at the center of the earth as

$$
\Omega=-r a^{3} H_{0} \sin \varphi / R^{3}
$$

where

$$
R=\left\{4(Z a)^{2}-4 r(Z a) \cos \phi \cos \lambda+r^{2}\right\}^{\frac{1}{2}},
$$

This gives the components of magnetic field strength at the ground surface

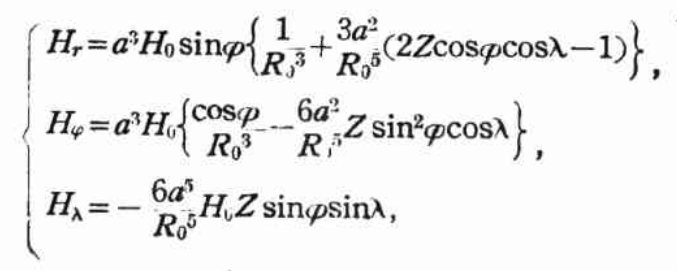


where

$$
R_{0}=a\left(4 Z^{3}-4 Z \cos \varphi \cos \lambda+1\right)^{\frac{1}{2}} .
$$

Thus we obtain the horizontal component of the disturbing field strength

$$
H^{\prime}=a^{3} H_{0}\left[\cos ^{2} \varphi / R_{0}^{3}-\left(12 a^{2} Z \sin ^{2} \phi \cos \varphi \cos \lambda\right) / R_{0}{ }^{5}\right.
$$

$$
\left.+\left\{36 a^{1} Z^{2} \sin ^{2} \varphi\left(1-\cos ^{2} \varphi \cos ^{2} \lambda\right)\right\} / R_{0}\right]^{-\frac{1}{2}} .
$$

Where $Z$ in not too small and the latitude not too high, $H^{\prime}$ shows a diurnal variation with a maximum at midday and a minimum at midnight. Fig. 8 represents the calculated diurnal variations of $H^{\prime}$ for several values of $Z$.

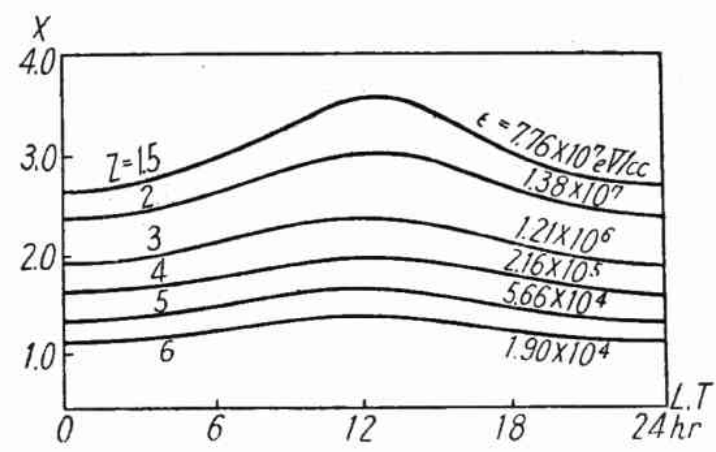

Fig. 8 The calculated diurnal variations of $\mathrm{H}^{\prime}$ for various values of $\mathrm{Z}$, when the face of the corpuscular stream is perpendiculır to the sun-earth line.

The above calculation is based on the assumption that the face of the stream is perpendicular to the sun-earth line. According to the recent suggestion by Prof. Ferraro, this is not the case, but the face of the stream will approach closest to the earth on the evening side of it, which we can also expect from the work of Prof. Alfvén [9]. We here report our results under correction from future investigators who will take account of these considerations.

\section{The magnetic shielding effect of the ionosphere}

To get a simple solution of this problem, we make the following approximation. The ionosphere is considered as a plain conductive plate with infinite extension, since the height and thickness of the ionosphere are small compared with the adius of the earth.

We approximate the real ionosphere by a set of numerous thin layers, each of which has a little different character from the neighbouring ones. A thin plate $(j)$ is piled up perpendicular to $x$-axis, and the boundary between plates $(j)$ and $(j+1)$ is denoted by $x_{j}$. $D_{j}=x_{j}-x_{j-1}$ represents the thickness of plate $(j)$ has a complex refractive index $p_{j}$ or characteristic impedance $q_{i}$, which are both expressed 
by dielectiric constant $\varepsilon_{j}$, magnetic permeability $\mu_{j}$, specific conductivity $\sigma_{j}$ and the period of electromagnetic wave $\tau$. These are

$$
\begin{aligned}
& p_{j}=\alpha_{j}-i \beta_{j}, \\
& q_{j}=\mu_{j} / p_{j},
\end{aligned}
$$

here

$$
\frac{\alpha_{j}}{\beta_{j}}=\left\{\begin{array}{c}
\mu_{j} \\
2
\end{array}\left(\sqrt{\varepsilon_{j}^{2}+4 \sigma \bar{j}^{2} \tau^{2}} \pm \varepsilon_{j}\right)\right\}^{\frac{1}{2}} .
$$

Considering that the electromagnetic wave propagates from negative to positive $x$, the electric and the magnetic field strength in the layer $(j)$ are expressed by

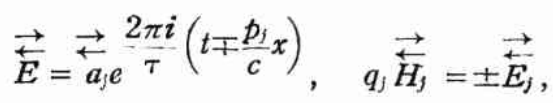

where $\rightleftarrows$ means the positive or negative direction of propagation. These waves must satisfy the boundary conditions. The continuity of tangential component of $E$ leads to

$$
\overrightarrow{a_{j} e}-(j, j)+\overleftarrow{a_{j} e}\left(\stackrel{(j, s)}{=} \vec{a}_{j+1} e^{-(j+1, j)}+\overleftarrow{a}_{j+1} e,^{(j+1, j)}\right.
$$

where

$$
e^{ \pm(j, k)}=e^{ \pm \frac{2 \pi i}{c \tau} p_{j} x_{k}}
$$

Similarly, the continuity of $H$ leads to

$$
q_{j+1} \overrightarrow{a_{j}} e^{-(j, j)}-q_{j+1} \stackrel{\leftarrow}{a_{j+1}} e^{(j, j)}=q_{j} \vec{a}_{j+i} e^{-(j+1, j)}-q_{j} a_{j+1} e^{(j+1, j)}
$$

We shall now define the complex ratios

$$
\boldsymbol{r}_{j, j+1}=\left(q_{j+1}-q_{j}\right) /\left(q_{j+1}+q_{i}\right) \text {, }
$$

and eliminating the component of $a$ from (5.5) and (5.7), we get

$$
\overrightarrow{a_{j}}\left(1+r_{j, j+1}\right) e^{-(j, j)}=\overrightarrow{a_{j+1}} e^{-(j+1, j)}+\vec{a}_{j+1} \boldsymbol{r}_{j, j+1} e^{(j+1, j)}
$$

and

$$
\overleftarrow{a_{j}}\left(1+r_{j, j+1}\right) e^{(j, s)}=\overrightarrow{a_{j+1}} \boldsymbol{r}_{j, j+1} e^{-(j+1, s)}+\overleftarrow{a_{j+}} e^{(j+1, s)}
$$

The reflective wave accompanied by such a penetrating wave should vanish behind the great number of layers, and we can write

$$
\overleftarrow{a_{s+1}} \equiv 0 \text {. }
$$

Therefore considering (5.5) and (5.7), we get

$$
\overleftarrow{a_{s}}=\overrightarrow{a_{s}} \boldsymbol{r}_{s,+i} e^{-2(s, s)} \text {. }
$$

Introducing this relation into (5.9) and (5.10), we have 


$$
\begin{aligned}
& \vec{a}_{s-1}\left(1+r_{s-1, s}\right)=\vec{a}_{s} e^{-(\Delta s-1, s-1)}\left\{1+r_{s-1}, r_{s, s+1} e^{-2(s, D s)}\right\}, \\
& \overleftarrow{a}_{s-1}\left(1+r_{s-1, s}\right)=\vec{a}_{s} e^{-(\Delta-1,-1)-2(s-1, s-1)}\left\{r_{s-1, s} r_{s, s+i} e^{-2(r, D s)}\right\},
\end{aligned}
$$

where

$$
e^{(j, D k)}=e^{(j, k)-(j, k-1)}, e^{(\Delta j, k)}=e^{(j+1, k)-(j, k)} .
$$

Thus we can express arbitrary $\underset{a_{\mu}}{\overleftrightarrow{\rightleftarrows}}$ with $\overrightarrow{a_{3}}$, by substituting the above relation into (5.9) and (5.10) successively. We can choose the thickness of the layer $D_{\text {; }}$ so thin that $\boldsymbol{r}_{j, j+1}<1$. Using $\left|e^{-(j, D j)}\right|<1$ and neglecting the second order of $\mathrm{r},(5.9)$ and (5.10) are reduced to

$$
\begin{aligned}
& \overrightarrow{a_{j}}=\overrightarrow{a_{j+1}}\left(1-r_{j, j+1}\right) e^{-(\Delta j, s)}+\overleftarrow{a_{j+1}} r_{j, j+1} e^{2(j, s)}, \\
& \stackrel{\leftarrow}{a_{j}}=\overrightarrow{a_{j+1}} r_{j, j+1} e^{-2(j, j)}+\overleftarrow{a_{j+1}}\left(1-r_{j, j+1}\right) e^{(\Delta, j)} .
\end{aligned}
$$

Suts tituting (5.11) into these formulae, we have

$$
\overrightarrow{a_{s-1}}=\overrightarrow{a_{s}\left(1-r_{s-1, s}\right)} e^{-(\Delta s-1, s-1)}, \stackrel{\leftarrow}{a_{s-1}}=\overrightarrow{a_{0} O}\left(r_{\delta-1, s}\right) .
$$

For any positive integer $\mu<\nu$, this is expressed as

$$
\overrightarrow{a_{\mu}}=\overrightarrow{a_{\nu}} \prod_{k=\mu}^{-1}\left(1-r_{k, k+1}\right)^{-\sum_{j=\mu}^{\nu-1}(\Delta f, j)},
$$

or in the limiting case $\Delta \rightarrow 0$ by the differential expression

$$
\overrightarrow{a_{\mu}}=\overrightarrow{a_{\nu}}\left(1-\frac{1}{2} \int_{x_{\mu}}^{x_{\nu}} d q\right) e^{-\frac{2 x_{i}}{\tau_{\tau}}} \int_{x_{\mu}}^{x_{\nu}} x d p .
$$

In the care $x_{\mu}=x$, we obtain

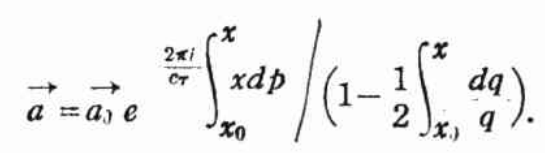

By this formula we can express the amplitude at any point $x_{\nu}$ by the incidental vave. Similarly, the reflexive wave is given by

$$
\overleftarrow{a_{\mu}}=\frac{\vec{a}_{\mu}}{2} \int_{x_{\mu}}^{x_{i}} \frac{e^{-{ }^{4 x_{\tau}}}\left(\int_{x_{\mu}}^{x} p d x-x_{\mu} p_{\mu}\right)}{\left.2 \int_{x_{\mu}}^{x} d q\right)} d q .
$$

Since the observed point lies on the ground surface in our case, we may put 


$$
q_{0}=q_{\nu} \simeq 1 .
$$

Further the conductivity $\sigma$ is finite everywhere, so that

$$
\boldsymbol{R}(q)>0
$$

then we have

$$
\oint \frac{d q}{q}=0 .
$$

Consequently, (5.19) is reduced to

$$
\overrightarrow{a_{\nu}}=\overrightarrow{a_{\nu} e^{-}}-\frac{2 \pi i}{c_{\tau}} \int_{x_{j}}^{x_{\nu}} x d p
$$

or by making use of (5.1)

$$
\overrightarrow{a_{\nu}}=\overrightarrow{a_{0}} e^{-\frac{2 \pi}{c \tau} \int_{x_{0}}^{x_{\nu}}\{\beta+i(\alpha-1)\} d x} .
$$

We can proceed with the calculation by the following simplification

$$
\beta=\beta_{\mathrm{G}} e^{-\frac{x^{2}}{4 D^{2}},} \quad \alpha=\beta+1,
$$

where $D$ represents the thickness of ionosphere and $\beta_{0}$ is related with the total conductivity $\sigma^{*}$ by

$$
\beta_{0}=\left(\frac{\tau \sigma^{*}}{\sqrt{2 \pi D}}\right)^{\frac{1}{2}}, \quad \sigma^{*}=\int \sigma d x .
$$

These are also expressed as

$$
\sigma=\sigma_{m} e^{-\frac{x^{2}}{2 D^{2}}}, \quad \beta=\sqrt{\sigma_{m} \tau} e^{-\frac{\partial^{2}}{4 D^{2}}},
$$

where $\sigma_{m}$ means the maximum specific conductivity. Substituting these quantities into (5.22), we obtain

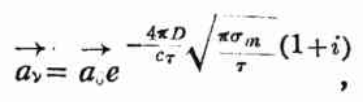

or

$$
\overrightarrow{a_{\nu}}=\overrightarrow{a_{1}} e^{-\frac{4 \pi}{c}\left(\frac{\pi}{2}\right)^{\frac{1}{4}} \sqrt{\frac{\sigma^{*} D}{\tau}}(1+i)}
$$

Further we must consider the induction effect by the earth crust, but here we may cnly assume that the shielding coefficient $S$ in the actual case is written as

$$
S=S^{\prime} /(1+\boldsymbol{r}),
$$

where $S^{\prime}$ is the shielding coefficient in the absence of earth crust and $r$ the mean reflection coefficient of the crust in this case, which we may put $r=1 / 3$ hereafter 
in numerical estimation. In Fig. 9 we rpresent $\log S$ as a function $\mathrm{cf} \log \sigma^{*}$, assuming that

$$
\tau=4 T=8 \times 10^{2} \text { sec., } D=120 \mathrm{~km}, r=1 / 3 .
$$

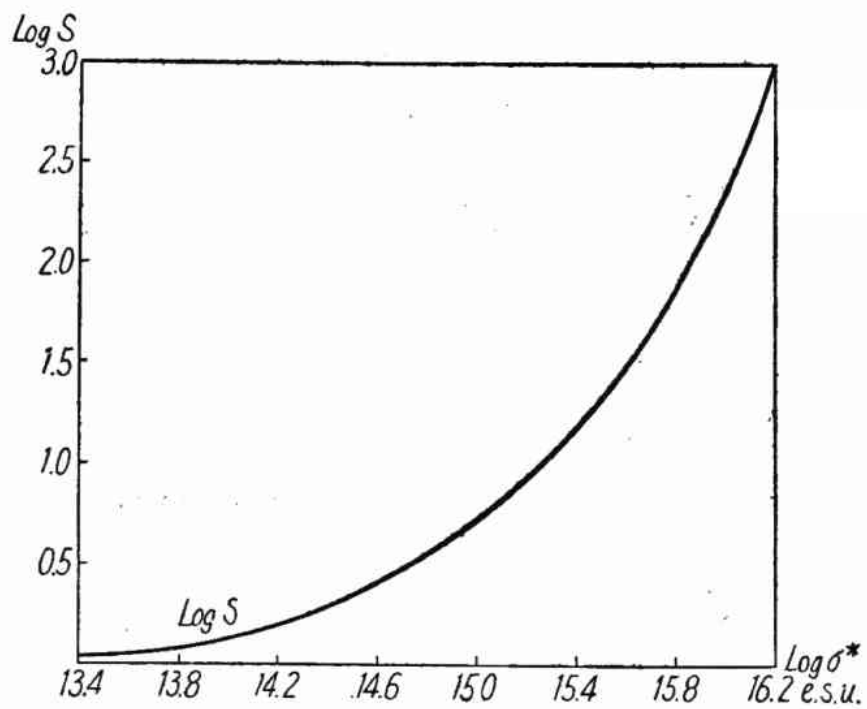

Fig. 9 The calculated shielding coefficient.

The shielding coefficient $S$ amounts to as large as $10^{3}$, when we adopt the total conductivity according to dynamo-theory, $\sigma^{*}=1.5 \times 10^{16}$ e.s.u. For this case, it leads to a very small increase of field strength, $H<3 \gamma$. Furthermore is expected an extraordinary large amplitude ratio of diurnal variation of $H$, owing to the large shielding ratio, $\mathrm{S}_{\text {nax }} / \mathrm{S}_{\text {nin }}=10^{2}$. These contradictions suggest that either the corpuscular hypothesis may not correspond to reality or the total conductivity, based on dynamo thcory, may not fit in with our case. [10] If we adhere to the corpuscular theory, a smaller value of $\sigma^{*} \simeq 6 \times 10^{14}$ e.s.u. would explain the observed fact. It is noticeable that this value is in agreement with that based on the radio wave observaion in icnosphere, $\sigma_{m}=2 \times 10^{7}$ e.s.u., $\mathrm{D}=120 \mathrm{~km}$. Such a situation suggests that the conductivity adequate to our problem may still be identical with the case of radio wave observation, though very different in frequensy. It will be very interesting if one could explain this feature fom the microscopic standpoint. Thus we get the shielding coefficient for this value of total conductivity,

$$
S \simeq 3.2 \text {. }
$$

The above result is not so inconsistent with the one by Sugiura [11] or by Ashour and Price [12], who treated the matter more satisfactorily and extensively with quasistationary method. I present here my approximate treatment of the shielding of the ionosphere in its original form because Ashour and Price's paper reached my hand after I had written this.

Following the above discussion, we take the diurnal variation of $\sigma^{*}$ as follows, 
taking into account both the results of radio wave observation and the dynamotheory by Chapman or Schusier.

$$
\sigma^{*}=\sigma_{0}^{*}\left(1+a_{1} \sqrt{\cos \kappa} \chi+a_{2} \cos \chi\right),
$$

where $\chi$ is the zenith angle of the sun, $a_{2}$ and $\sigma_{0}^{*}$ are the constants determined by comparison with actual observation. $a_{1}$ is such a constant that

$$
\begin{array}{rlrl}
a_{1} & \neq 0 & \text { if } & |\chi| \\
& =0 & & >\pi / 2 \kappa, \\
& >\pi / 2 \kappa,
\end{array}
$$

here $\kappa$ serves to represent the effect of $\sqrt{\cos \chi}$ in the night hemisphere and, ther fore, $\kappa<1$.

Substituting (5.31) into (5.27) and (5.28), we obtain the shielding coefficient as

$$
S=\underset{1+r}{1} \exp \left\{\frac{4 \pi}{c}\left(\begin{array}{c}
\pi \\
2
\end{array}\right)^{\frac{1}{4}} \sqrt{\frac{D \sigma_{0}^{*}}{\tau}}\left(1+a_{1} \sqrt{\cos \kappa \chi}+a_{2} \cos \chi\right)^{\frac{1}{2}}\right\} .
$$

\section{Diurnal variation of $\mathbf{H}$}

Making use of the results obtained in the preceding two sections, we can explain the observed diurnal variation of $\mathrm{H}$. In the Equinox, the zenith angle of the sun is given by

$$
\cos \chi=\cos \phi \cos \lambda \text {. }
$$

Here the angle $\lambda$ is expressed by local time $T_{L}$ as

$$
-\cos \lambda=\cos \left(\pi T_{L} / 12\right) \text {. }
$$

Hence the diurnal variation of $\mathrm{H}$ is determined by equations (3.5), (4.4), (5.32) and (6.1) or (6.2).

In Fig. 3, the full line is drawn by these formulae corresponding to the case

$$
D=120 \mathrm{~km}, \quad \tau=800 \mathrm{sec} ., \quad \sigma^{*}=6 \times 10^{14} \text { e.s.u. }
$$

and

when

$$
\stackrel{*}{\sigma}_{\max } / \stackrel{*}{\sigma}_{\min }=5, \quad a_{1} / a_{2}=2, \quad \kappa=2 / 3,
$$

$$
\varepsilon=3 \times 10^{5} \quad e V / c c
$$

at the place $p=36^{\circ}$. We can say that the calculated curve of $\mathrm{H}$ is in good agreement with the observational one. For various values of parameters, we get the curve in Fig. 10, in reference to the case at the equator. The curves for $B$ and $C$ groups defined in section 2 are also in good agreement with the case of $\varepsilon=10^{\circ} \sim 10^{\circ}$ $e V / c c$. Now we come to the question left unsolved in section 3. To derive (3.11) we assumed that $Z$ is not too near to unity. This condition is really fulfilled as discussed in what follows. Even if this condition is not satisfied, the order of magnitude of $\varepsilon_{2}$ and $\varepsilon_{1 m}$ does not change. Adopting (5.30) in the value of $S, \varepsilon$ is the order of 

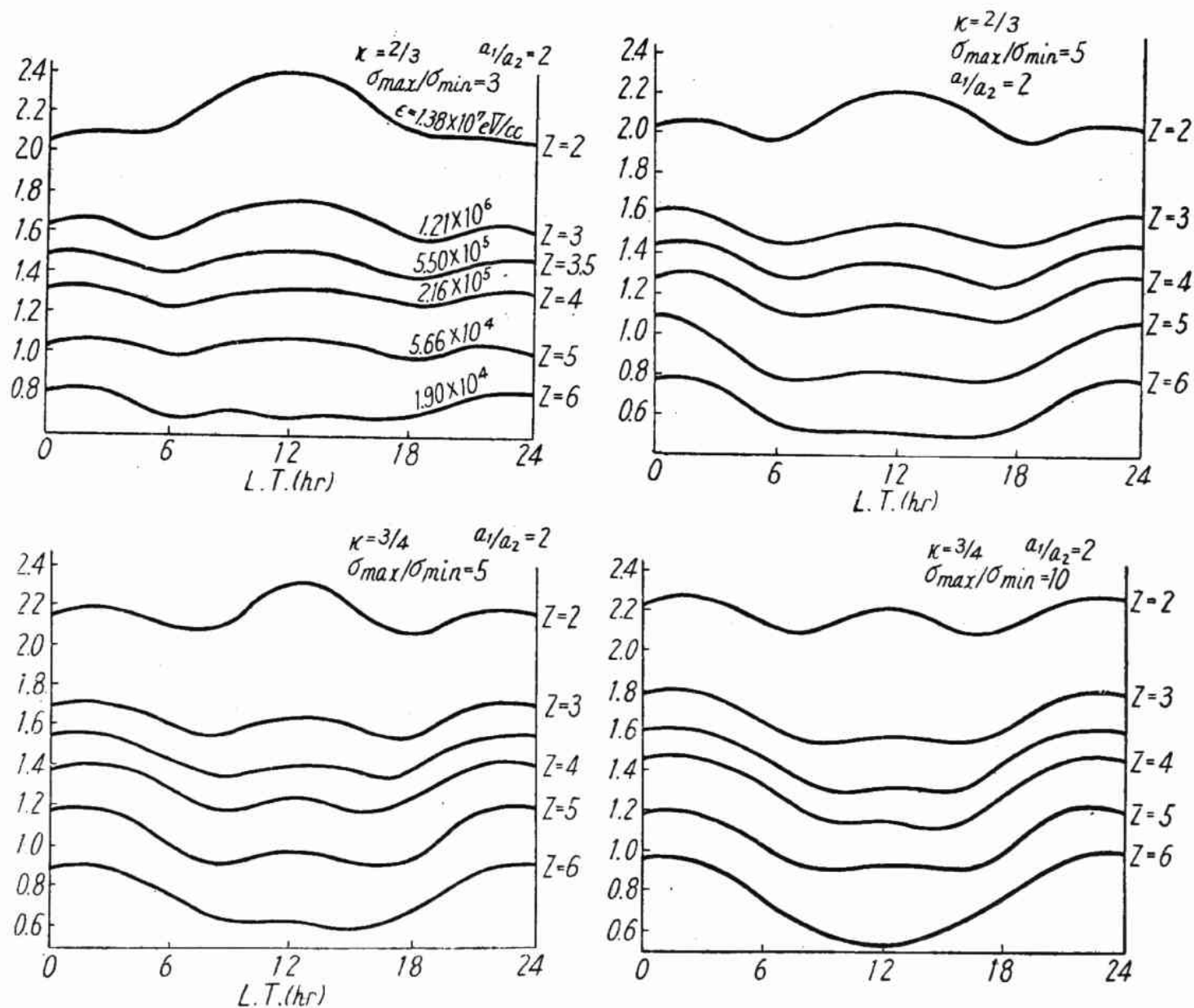

Fig. 10 The calculated curves of the diurnal variation of $\mathrm{H}$, taking account of both spatial distribution of $\mathrm{H}^{\prime}$ and the shielding effect of the ionosphere.

$10^{-7} \mathrm{erg} / \mathrm{cc}$. Considering (3.2), this value correponds to $Z=3 \sim 4$, which actually. fulfills the above condition. As for the average kinetic energy density we obtain

$$
\varepsilon \simeq 2.6 \times 10^{5} \mathrm{eV} / \mathrm{cc}
$$

by a simple calculation.

\section{Fimission mechanism of corpuscular masses}

Acoording to the statistical result concerning the correlation of sun-spot and magnetic storm, it seems that magnetic storms occur about $3 \sim 4$ days later than the sun-spot maximum. This is the very reason why the storm is considered to be caused by corpuscle, having lower velocity than that of photon, even though the time lag is a little ambiguous in the light of statistics. Here we use the values for the average velocity of the corpuscular masses as

$$
v=10^{i} \sim 10^{8} \mathrm{~cm} / \mathrm{sec} .
$$


The radius $\mathrm{A}$ of such masses may be

$$
A=1.5 \times 10^{12} \mathrm{~cm},
$$

assuming that the mean velocity is $5 \times 10^{7} \mathrm{~cm} / \mathrm{sec}$ and considering that the most disturbing period appears near the tail of a corpuscular mass.

Considering $\varepsilon=\nu m v_{0}^{2} / 2$, where $m$ is the mass of corpuscle and $\nu$ its molecular density near the earth, and assuming that the corpuscules are $\mathbf{C a}$ atoms, we get

$$
\nu=4.9 / c c \text {. }
$$

Then the average kinetic energy per each corpuscle is

$$
\bar{\varepsilon}=5.3 \times 10^{4} \mathrm{eV}
$$

and the total mass of a corpuscular mass is

$$
M=4.6 \times 10^{9} \text { tons. }
$$

Next we derive $\delta$ from the formula

$$
\delta=\nu k T / p,
$$

where $p$ and $T$ mean respectively the pressure and the temperature of a corpuscular mass near the sun's surface. These are equated to the value at the sunspot

$$
p=10^{5} \text { c.g.s., } \quad T=4000^{\circ} \mathrm{K} \text {. }
$$

Then we have

$$
\delta=2.7 \times 10^{-18}
$$

which is not inconsistent with Chapman's supposition. [13] From (7.2) and (7.7), the average radius of a corpuscular mass in the first step $A_{1}$ is taken as

$$
A_{1}=21 \mathrm{~km}
$$

According to (3.14), (3.15), (5.30), and (7.7)

$$
\begin{aligned}
& \varepsilon_{2}=1.37 \times 10^{10} \mathrm{erg} / \mathrm{cc}, \\
& \varepsilon_{1 m}=1.52 \times 10^{11} \mathrm{erg} / \mathrm{cc} .
\end{aligned}
$$

Based on the constants obtained above, we shall again discuss the hypotheses in section 3. There we assumed that the sun is a turbulent fluid system in which the transportation of energy is mainly due to a mass unit of ionized corpuscles which would cause a magnetic storm to occur. If this is the case, $\varepsilon_{1 m}$ should have a close connection with its temperature. For the sum of thermal and mechanical fotential energy may approximately be conserved since the sun is considered to be in radiation equilibrium. Further, the contribution of potential energy may be neglected, so long as our consideration is restricted near the surface of the sun. Consequently the kinetic energy of turbulent motion may be converted mainly from the thermal energy in the initial state. This may explain the low temperature of the sun-spot, which is considered as a violent turbulence on the solar disc. Alti o tgh we cannot get any reliable and detailed knowledge concerning, say, what portion $n f$ 
the thermal energy in initial state is converted into the energy of mass motion, or how to determine the constant corresponding to Boltzmann's in this case, etc., it is reasonable to assume that the emission mechanism of corpuscular masses described above is tolerably true to fact, considering that not every sun-spot corresponds with some one magnetic storm, though there is a high correlation between them, and that the correlation between magnetic storm and sur-spot in higher latitudes is undeniable. That is why we speak of corpuscle "mass" and not of "slab stream".

The author wishes to acknowledge his indebtedness for the materials used in this investigation and for important suggestion to Dr. S. Imamichi, chief of Kakioka Magnetic Observatory, and to Dr. $O$. Minagawa, director of the Laboratory of Electromagnetism, Meteorological Research Institute, for continued support and encouragement, and Mr. S. Hayakawa, who generously gave helpful advice and stimulus in his conversation with the present author.

\section{References}

[1] F.A. Lindemann: Phil. Mag. 33, 669 (1919)

[2] E.A. Milne: Mon. Not. R. Astr. Soc. 86, 459, 578 (1926)

[3] M. Notuki: Report of Ionosphere Research in Japan, 4, 104 (1950)

[4] C. Störmer: Terr. Mag. 22, 97 (1917)

[5] C. Störmer: Terr. Mag. 35, 193 (1930)

[6] S. Chapman and V.C.A. Ferraro: Terr. Mag. 36, 77, 171 (1931), 37, 147, 421 (1932), 38, 70 (1933), 45, 245 (1940)

(7) M.S. Vallarta and O. Godart: Rev. of Mod. Phys., 11, 180 (1939)

[8] T.G. Cowling: Terr. Mag. 47, 209 (1942)

[9] H. Alfveen: Kungl. Svenska Vet. Ak:s, Handl. III. Bd. 18, NO 3, (1939);

Bd. 13, N:O 20, (1940)

[10] S. Chapman: Phil. Trans. Roy. Soc. London, 218, 1 (1919)

[11] M. Sugiura: Report of Ionosphere Research in Japan, 3, 55 (1949)

[12] Ashour and Price: Proc. Roy. Soc., A, 195, 198 (1948)

[13] S. Chapman and V.C.A.Ferraro: Mon. Not. R. Astr. Soc. 89, 470 (1929). 


\section{THE FOURTH RESEARCH MEETING}

OF

\section{THE METEOROLOGICAL RESEARCH INSTITUTE}

The fourth research meeting was held at the Central Meteorological Observatory for three days, the 29th, the 30th, and the 31st of March, 1950. The authors and the subjects of papers read there are as follows:-

The 1st day (Mir. 29th, 1950)

1. T. Fujita: A Note on the Correlation.

2. M. Ogawara: Study on Stochastic Forecasting(VI) On Testing of Serial Correlation Coefficient.

3. M. Masuyama : On Approximate Latent Values of Quadratic Pairs.

4. M. Sanuki \& S. Agari: On Computation of Coordinates Conrespondency of Conformal Representation.

5. T. Ishimori : On Correlations between Solar Constant and Temperature.

6. S. Oazawa: Statistical Study on Agricalture-meteorological Accident in Japan.

7. T. Yazawa: On Climatic Landscape (Some otserved data about climatic environment of Tenryu-valley, Nagano Prefecture).

8. T. Sekiguchi: On New Method of Classification of Climatic Regions and Significance of Climatic Divide.

9. H. Maruyama: Studies on Fire.

10. Applied Meteorological Laboratory \& Public Health Institute: Study of Dwelling Condition Adept for Climate.

11. M. Momiyama: On Distribution Curve of Seasonal Disease.

12. T. Kashiwagi \& M. Masuyama: On the Electro-chromatographic Analysis of So-called Fatigued Person's Urine.

13. N. Zennyoji \& E. Suzuki: On Type of Precipitations.

14. I. Imai : On Fall Velocity of Raindrops.

15. G. Yamamots \& A. Yamamoto: On Evaporation from Large Water-surface.

16. Physical Meteorological Laboratory \& Geochemical Laboratory: Physical and Chemical Research on River Fogs.

17. Y. Miyake \& Y. Sugiura: On Chemical Constituents Dissolved in Rain Water Accompanied by Typhoons.

18. Y. Miyake \& Y. Sugiura: On Mechanism of Dissolution of Saline Matter into Rain Water.

19. Y. Miyake \& K. Saruhashi : On Diss lution of Oxygen into Rain Drop.

20. K. Soma, Y. Kawano, H. Nishiyama, S. Iwase \& T. Watanabe: On Error Caused by Moment Inertia of Cup Anem meter in the Changing Wind.

21. K. Tsukamoto \& N. Tsuneska : On Pressure-type Remoto Rain Gage.

22. K. Tsukamoto \& N. Tsunecka: Research on Telemetering Rain Gage.

23. M. Sanuki, H. Kamamoto \& S. Kimura : Research on New Combination Modes of Wind Vane and Anemometer (The 2nd report).

24. H. Kamamoto \& S. Kimura: Research on Wind Speed and Direction Indicating Robot for Isolated Islands Use.

25. K. Isono \& E. Hayashi: On Application of Thermistors ts Meresrological Observation (1).

The 2ad day (Mar. 30th, 1953)

26. M. Okuta: Westerly Troughs on $700 \mathrm{mb}$ Level of Northern Hemisphere (I).

27. E. Suzuki \& T. Ishimori: On Correlation Coefficients on Seasonal Forecasting. 
28. M. Ida \& T. Watanabe: Annual Variation of Pressure Gradient in Japan Area.

29. N. Nakamura \& T. Watanabe: On Seasonal Climatic Variation (2): On Separation of Disturbances from Basic States.

3n. K. Osawa: On Change of Normal Broad Weather Situation.

31. K. Takahashi: On Method of Seasonal Forecasting due to Periodogram Analysis.

32. K. Takahashi: On Average of Meteorological Elements.

33. K. Takahashi: On Weather Proverbs Concerning Seasonal Forecasting.

34. S. Oi: On Upper Air Advection and High and Low.

35. S. Oi: On Eend of Isobars caused by the Central Mountains of Japan.

36. M. Itokazu: Summer Upper Air Analysis.

37. M. Takeuchi \& M. Itokazu: On Influences of Perturbations in Westerlies in Low Latitudes and Thunderstorms at Kanto District.

38. T. Fujita: On Upper-air Weather Chart of the Time Typhoon "Della" passed.

39. T. Fujita: On Errors in Determination of Isobaric Surface Height from Observations by Radio-Sondes Currently in Use.

4n. S. Kubota: On Typhoon "Kitty".

41. T. Ando: On Rains Acompanied by Typhoon.

42. M. Magata: On Eye of Storm.

43. T. Watanabe: Dynamical Study on Mechanism of Convergence.

44. T. Izawa: On Upper Disturbances in Westerlies having Longitudinal Wind Gradient.

45. H. Arakawa: On Zonal Oscillations of Atmosphere and Their Stability.

46. S. Matsumoto: On Upper Wave and Movement of (Anti-) Cyclone.

47. M. Takeuchi: Studies of Heat Convection (The 3rd report).

48. M. Takeuchi: Studies of Fohn Phenomena (The 3rd report), On the Lohn Fow.

The 3rd day (Mar. 31st, 1950)

49. N. Saito: On Eddy Turbulence in Free Atmosphere.

5n. S. Matsumoto: A Proposition fur Problem of Advection.

51. Y. Masuda: On Computing Method of Vertical Velocity in Atmosphere (The 3rd repcrt).

52. Y. Masuda: On Dynamics of Baroclinic Disturbance in Upper Troposphere.

53. K. Isono \& Y. Hujiwara: On Code-sending type Ratiosonde (III).

54. K. Isono, Y. Fujiwara \& Y. Toyama: On Code-sending type Radiosonde (IV).

55. N. Arizumi: On Analysis of Aerology over Japan and its Neighbourhood during the Term from the 8th to the 18th of December, 1919 (The Second International Aerological Days in Winter).

55. K. Sekihara : $\Lambda$ Study on Distribution of Ultraviolet Sky Radiation.

57. Y. Miyake \& K. Saruhashi: On Annual an 1 Latitudinal Variation of Ozone Layer.

58. S. Unoki: On Time-lag of Prutuberance of Surface owing to Depression.

59. J. Sugiura: On Ocean Current in Antarctic Ocean.

60. S. Fukase : On Distribution of Dissolved Oxygen in Okhotsk Sea.

61. M. Nagai : On Diurnal Variation of Surface Layer Tem: erature of Lake Chuzenji-ko in Autumn.

62. G. Fujioka, I. Kita \& O. Minagawa: $\Lambda$ Note Method of G. M. Counter Construction.

63. G. Fujioka, I. Kita \& O. Minagawa: On Cosmic-ray Hoduscope.

64. G. Ishikawa: On Jlectromagnetic Wave Passing through Non-uniform Conductor and its Application to the Theory of $\mathrm{Ma}_{5} \mathrm{n}$ tic Storm (Supplement). 